\title{
Sensory integration dysfunction affects efficacy of speech therapy on children with functional articulation disorders
}

This article was published in the following Dove Press journal:

Neuropsychiatric Disease and Treatment

16 January 2013

Number of times this article has been viewed

\author{
Li-Chen Tungl,\# \\ Chin-Kai Lin ${ }^{2, \#}$ \\ Ching-Lin Hsieh ${ }^{3,4}$ \\ Ching-Chi Chen' \\ Chin-Tsan Huang' \\ Chun-Hou Wang 5 \\ 'Department of Physical Medicine \\ and Rehabilitation, Chi Mei Medical \\ Center, Tainan, ${ }^{2}$ Program of Early \\ Intervention, Department of Early \\ Childhood Education, National \\ Taichung University of Education, \\ Taichung, ${ }^{3}$ School of Occupational \\ Therapy, College of Medicine, \\ National Taiwan University, Taipei, \\ ${ }^{4}$ Department of Physical Medicine \\ and Rehabilitation, National Taiwan \\ University Hospital, Taipei, ${ }^{5} \mathrm{School}$ \\ of Physical Therapy, College of Medical \\ Science and Technology, Chung Shan \\ Medical University, Taichung, 'Physical \\ Therapy Room, Chung Shan Medical \\ University Hospital, Taichung, Taiwan \\ \#These authors contributed equally
}

Correspondence: Chun-Hou Wang School of Physical Therapy, College of Medical Technology, Chung Shan Medical University, No II 0 , Sec I, jianguo N Rd, Taichung City 4020I, Taiwan

Tel +886-4-24730022 ext I 2390

Fax +886-4-24759950

Email chwang@csmu.edu.tw
Background: Articulation disorders in young children are due to defects occurring at a certain stage in sensory and motor development. Some children with functional articulation disorders may also have sensory integration dysfunction (SID). We hypothesized that speech therapy would be less efficacious in children with SID than in those without SID. Hence, the purpose of this study was to compare the efficacy of speech therapy in two groups of children with functional articulation disorders: those without and those with SID.

Method: A total of 30 young children with functional articulation disorders were divided into two groups, the no-SID group (15 children) and the SID group (15 children). The number of pronunciation mistakes was evaluated before and after speech therapy.

Results: There were no statistically significant differences in age, sex, sibling order, education of parents, and pretest number of mistakes in pronunciation between the two groups $(P>0.05)$. The mean and standard deviation in the pre- and post-test number of mistakes in pronunciation were $10.5 \pm 3.2$ and $3.3 \pm 3.3$ in the no-SID group, and $10.1 \pm 2.9$ and $6.9 \pm 3.5$ in the SID group, respectively. Results showed great changes after speech therapy treatment $(\mathrm{F}=70.393$; $P<0.001)$ and interaction between the pre/post speech therapy treatment and groups $(\mathrm{F}=11.119$; $P=0.002)$.

Conclusions: Speech therapy can improve the articulation performance of children who have functional articulation disorders whether or not they have SID, but it results in significantly greater improvement in children without SID. SID may affect the treatment efficiency of speech therapy in young children with articulation disorders.

Keywords: children, functional articulation disorders, sensory integration dysfunction, speech therapy, efficacy

\section{Introduction}

Articulation refers to the totality of motor processes involved in the planning and execution of speech. Articulation disorders are difficulties with the motor production aspects of speech, or an inability to produce certain speech sounds. ${ }^{1}$ Possible causes of articulation disorders including problems affecting motor production centers (ie, tongue/ palate/muscular), sensory feedback centers (ie, auditory, vestibular, proprioceptive, tactile), generalized central nervous system (CNS) processes (ie, developmental delay), and focal CNS processes (ie, stroke, cerebral palsy, or tumor affecting receptive and expressive speech centers, and motor production areas). ${ }^{2}$ If all the structures required for speech production and articulation are intact, but a child has difficulty learning to make a specific speech sound for no clear reason, the child is considered to have a functional articulation disorder. ${ }^{1}$ 
Functional articulation disorders are common in young children. ${ }^{3}$ A 1994 report of the National Institute on Deafness and Other Communication Disorders estimated that approximately $10 \%$ of the population is affected by phonological disorders (including articulation disorders). ${ }^{3}$ In the US, Shriberg and Kwiatkowski proposed that $7.5 \%$ of all children aged 3-11 years experienced articulation disorders. ${ }^{4}$ As for the prevalence of functional articulation disorders, evidence from the literature indicates rates of $1 \%-21 \%$. A conservative estimate of the prevalence rate in preschool children is approximately $10 \%{ }^{5}$

Sensory integration means that an individual transmits information with regard to contact between the body and the surrounding environment via one or more sensory systems (ie, the visual, auditory, tactile, gustatory, olfactory, vestibular, and proprioceptive) to the brain for integration, and then the brain tells the motor system to respond. ${ }^{6}$ Sensory integration theory is used to explain mild-to-moderate issues in learning and behavior, especially movement coordination and sensory modulation dysfunction, as well as problems not caused by central nervous injury or abnormality. ${ }^{7,8}$ Sensory and motor experiences are the foundation of speech development. Many young children with speech disorders have reduced functions in the vestibular, proprioceptive, and tactile sensory systems compared to normal children. ${ }^{9}$ When a child has defects in sensory systems such as the auditory perception or vestibular system, speech development will be affected, causing problems such as delays in speech development and articulation disorders. Ayres hypothesized that sensory integration dysfunction(SID) is related to the processing ability of the central sensory system. ${ }^{7}$ A diagnosis of SID requires evidence of defects in the vestibular, proprioceptive, or tactile sense not caused by central or peripheral nerve damage or cognitive deficit. ${ }^{8}$ In terms of the mode of articulation disorder, McDonald believed that articulation disorders in young children were due to defects occurring at a certain stage in sensory and motor development. ${ }^{10}$ That report emphasized that the most fundamental issue of articulation disorders lies in defects in sensory and motor development. Sensory integration involves the ability to perform motor actions after integrating sensory input. Therefore, some children with functional articulation disorders may also have SID.

A study by Gierut showed that most children with articulation disorders showed good improvement after proper therapeutic interventions, including: (1) the sensory-motor method of sound teaching; (2) cycles: auditory bombardment in conjunction with sound production; (3) minimal pair treatment: two words that differ by one sound; and (4) metaphon: emphasis of contrasts among speech sounds and sound properties. ${ }^{3}$ Many researchers have found that children with articulation disorders also have concomitant deficits in fine motor coordination and attentiondeficit/hyperactivity disorder (ADHD). ${ }^{11-13}$ SID can cause deficits in fine motor coordination and ADHD. ${ }^{14}$ However, whether SID reduced the efficacy of speech therapy on children with functional articulation disorders is unknown. Hence, the purpose of this study was to compare the efficacy of speech therapy in two groups of children with functional articulation disorders: those with and those without SID. We hypothesized that speech therapy would be less efficacious in children with SID than in those without SID.

\section{Materials and methods \\ Participants}

The study subjects were recruited through the rehabilitation clinic of a medical center in southern Taiwan. Based upon the findings of two previous studies defining the suitable age range for speech therapy intervention in preschool children, ${ }^{15}$ the inclusion criteria included: (1) diagnosis of functional articulation disorder; and (2) aged 42-72 months. The exclusion criteria included the following three conditions: (1) no developmental delay after clinical assessment and screening with the Comprehensive Developmental Inventory for Infants and Toddlers diagnostics and screening test (CDIIT), ${ }^{16}$ such that those with suspected gross motor, fine motor, or speech delays were excluded; (2) no hearing abnormality, tongue-tie, cleft lip and palate, other abnormal neurological findings, developmental apraxia of speech, or cognitive impairment; and (3) no previous receipt of speech therapy, or receipt of speech therapy in another hospital which had stopped for at least 3 months. We excluded subjects who visited other hospitals for speech therapy while receiving the therapeutic intervention in this study. This study was approved by the hospital Institutional Review Board and the study complied with the tenets of the Declaration of Helsinki. Before inclusion, the parents of the subjects fully understood the objective of the study, agreed to participate in the study, and signed the informed consent.

A total of 35 children were screened for inclusion. Three children were excluded due to developmental delay, and another two due to cognition impairment. The study was designed prospectively, and a total of 30 children were enrolled in this study. The no-SID group had 15 children ( 5 boys and 10 girls) and the SID group had 15 children ( 8 boys and 7 girls).

\section{Procedures}

For children who fit the inclusion criteria, the following assessments were performed before therapeutic intervention: (1) clinical articulation function evaluation, and (2) the Sensory Integration Functions Assessment Scale (SIF) to 
assess sensory integration function. Based on the results of the SIF, subjects were divided into two groups. Those who scored below 85 (out of 100) were assigned to the no-SID group; those who scored 85 and above were assigned to the SID group. After group assignment and parental agreement to comply with the therapy, therapeutic intervention began. The speech therapist who performed the Clinical Test of Articulation was not informed of the group assignments or the purpose of this study.

\section{Intervention protocols}

The basic principles of speech therapy for functional articulation disorders include: (1) auditory discrimination; (2) correct pronunciation of the target sound; (3) stability in producing the target sound; and (4) ability to generalize the target sound in different situations. ${ }^{2}$ The speech therapy was one-on-one, 30-minute sessions held once a week for 3 consecutive months. The speech therapist who administered therapeutic intervention was not the speech therapist who administered the assessments.

\section{Study tools}

Comprehensive Developmental Inventory for Infants and Toddlers diagnostics and screening test

The CDIIT is a standardized developmental test used to determine developmental delay in infants in Taiwan. ${ }^{16}$ It is used for infants aged 3-71 months to evaluate five major developmental areas: cognition, language, motor development, social development, and self-care ability. The test includes two sets of questions for diagnosis and screening. Up to 3703 infants and young children in Taiwan were used as the standardized sample to establish the diagnosis test, including the overall development and specific development according to age score, percentile, and developmental quotient in 30 age groups, as well as the $z$-score of seven age groups given the screening test. The test involves two types of assessment - direct testing of the child and parental completion of a questionnaire - to collect data on young children's development and behavior. The 343 questions in the diagnosis test have a test-retest reliability coefficient of 0.09-0.99; the observer reliability coefficient is between 0.19 and 0.56 ; the coefficient of internal consistency is between 0.75 and 0.99 . In validity testing, the correlation coefficient between each subtest and the overall score is between 0.96 and $0.99 .{ }^{16}$ Rasch analysis was used to analyze the CDIIT developmental screening test to determine the 87 most "suitable" and most easily implemented test questions for each corresponding developmental area within each age group. The correlation between subtests and overall score is $0.96-0.98$, indicating the high accuracy of both the screening test and the diagnosis test. In this study, the screening test was used to exclude cases with developmental delay. ${ }^{16}$

\section{The Sensory Integration Functions Assessment Scale} The SIF was developed in 2004. ${ }^{17}$ This scale assesses sensory integration using seven subscales: postural movement, bilateral integration sequencing, sensory discrimination, sensory modulation, sensory searching, attention and activity level, and emotional behavior. The scale contains 98 questions, which take approximately 20 minutes for a main caregiver to answer. The evaluator must have been the main caregiver for at least 6 months. Children aged 3-6 years are given the preschool version of the scale, while children aged 7-10 years are administered the school-age version of the scale. Each question is scored on a five-point Likert-like scale, for a total score of 98-490 points. Higher scores indicate poorer sensory integration function, while lower scores indicate sensory integration function approaching normal. The original sum score can be converted into a percentile rank based on the norm reference table. A percentile rank value $<72$ is considered normal, while percentile ranks between 73 and 84 are considered to show poor integration adjustment. Percentile ranks between 85 and 94 indicate borderline disorder, and a percentile rank $\geqq 95$ shows a disorder. This research tool has high test-retest reliability (0.87), and the all subscales have acceptable construct validity. ${ }^{17,18}$

\section{Clinical Test of Articulation}

In this test, an appropriate vocabulary set is used to evaluate the 21 individual consonants of the Chinese language. To verify the objectivity of the results, reliability and validity testing were performed using 10 young children with functional articulation disorders. Each child was tested separately with the Clinical Test of Articulation and the Mandarin Consonant Articulation Test for Preschool Children. ${ }^{19}$ Two speech therapists with more than 3 years of experience interpreted the tests. One therapist utilized both tests for evaluation; the other used only the Clinical Test of Articulation. The results of numbers of mistakes in pronunciation were compared with those of the Mandarin Consonant Articulation Test for Preschool Children for examining criterion-related validity. Interrater reliability was verified with the intraclass correlation coefficient (ICC). The ICC value obtained (0.93) met the standard of excellence. Criterion-related validity was tested using Spearman's correlation coefficient; the result (rho $=0.96$ ) supports the validity of the Clinical Test of Articulation. 


\section{Data analysis}

This study utilized SPSS for Windows (version 14.0; IBM, Armonk, NY, USA) for data analysis. Independent $t$-test and Chi-square test were used to test the difference in age, pretest number of mistakes in pronunciation between the two groups, sex, sibling order and education of parents. One-way repeated measures ANOVA was used to examine the difference in the mean number of mistakes in articulation in the two groups before and after treatment. A $P<0.05$ indicated statistical significance. The Cohen's $d$ was calculated to evaluate the effect size of the difference.

\section{Results}

Table 1 presents the characteristics of the no-SID and SID groups. A total of 30 subjects were recruited, and each group had 15 children. There were no statistically significant differences between the two groups in age, sex, sibling order, education of parents, or pretest number of mistakes in pronunciation $(P>0.05)$. These results demonstrated the homogeneity of the two groups before speech therapy.

The mean and standard deviation in the pre- and post-test number of mistakes in pronunciation in the no-SID group were $10.5 \pm 3.2$ and $3.3 \pm 3.3$, and in the SID group, $10.1 \pm 2.9$ and $6.9 \pm 3.5$, respectively. One-way repeated measures ANOVA indicated that the $P$ value of the treatment efficacy was $\leqq 0.001$. For treatment by group interaction, the $P$ value was 0.002 (Table 2). The effect size of the no-SID group was 2.22, while that of the SID group was 1.00. This difference indicated that no-SID group had a larger improvement in the number of mistakes in pronunciation than the SID group.

\section{Discussion}

This study compared the efficacy of speech therapy in two groups of children with functional articulation disorders: those with and those without SID. No demographic differences were

Table I Demographic and baseline data of participants

\begin{tabular}{llll}
\hline & Group & P value \\
\cline { 2 - 3 } & $\begin{array}{l}\text { No-SID } \\
(\mathbf{n}=15)\end{array}$ & $\begin{array}{l}\text { SID } \\
(\mathbf{n}=15)\end{array}$ & \\
\hline Age, months & & & \\
$\quad$ Mean (SD) & $58.9(7.6)$ & $57.5(7.7)$ & $0.620^{\mathrm{a}}$ \\
Sex (boy/girl) & $10 / 5$ & $8 / 7$ & $0.456^{\mathrm{b}}$ \\
Sibling order I/2/3 & $5 / 8 / 2$ & $6 / 7 / 2$ & $0.924^{\mathrm{b}}$ \\
$\begin{array}{l}\text { Education of parents } \\
\quad \text { Junior/senior high school/college }\end{array}$ & $2 / 12 / 1$ & $0 / 15 / 0$ & $0.189^{\mathrm{b}}$ \\
$\begin{array}{l}\text { Number of mistakes in pronunciation } \\
\quad \text { Prespeech therapy, mean (SD) }\end{array}$ & $10.5(3.2)$ & $10.1(2.9)$ & $0.683^{\mathrm{a}}$ \\
\hline
\end{tabular}

Notes: andependent $t$-test; ${ }^{b} \mathrm{Chi}$-square test.

Abbreviations: SD, standard deviation; SID, sensory integration dysfunction. found between the two groups. Both groups showed significant improvement in the pre- and post-test number of mistakes of pronunciation $(P<0.001)$. Comparison between groups demonstrated that children without SID showed significantly greater improvement. SID may account for mild-to-moderate problems in learning and behavior, caused by SID directly affecting the cerebral learning process or interfering with learning due to poor behavior. ${ }^{20}$ These principles and the study results suggest that SID can cause learning inefficiency and consequent delays in the articulation progress.

The treatment method in this study was a weekly, half-hour one-on-one session. The course of treatment included 12 treatment sessions over 3 months. Once the 3-month period passed, subjects were followed up to check the therapeutic efficacy. The therapy model in this study was developed based on many studies, including that by Rvachew et al, ${ }^{21}$ which found that children with articulation disorders had poorer recognition of phonological systems. After 16 sessions of phonological training, both phonological recognition and pronunciation accuracy improved. ${ }^{20}$ Forrest and Iuzzini found good therapeutic efficacy for a 20 -session treatment protocol of 1-2 therapy sessions per week, 20-30 minutes per session. ${ }^{22}$ A study involving the strategy of selecting target sounds to learn pronunciation found that training in related target sounds significantly improves pronunciation clarity more than random training. ${ }^{23}$ Another study involving the strategy of sound discrimination and production training reciprocity showed it to be a more effective procedure for correcting articulation. ${ }^{24}$ To summarize past studies of therapeutic efficacy, children with articulation disorders improve after receiving speech therapy with a treatment protocol of once or twice per week, half an hour per session. The course of treatment should last 12 to 20 sessions, or 10 weeks to 3 months, to achieve significant therapeutic efficacy. The methods for correction can be sound discrimination, target sound training, phonetic placement or oral-motor functional training. ${ }^{25,26}$ The treatment protocol in this study followed the above model and the results indicated significant efficacy.

In the theoretical framework developed by Oetter et al, ${ }^{20}$ in terms of the related development of suck/swallow/ breathe synchrony, articulation is at the outermost ring of sensorimotor development. This indicates that articulation has a certain correlation with the developmental process of sensory integration. ${ }^{20,27}$ Nasir and Ostry ${ }^{28}$ suggested that, during the process of sound production, in addition to speech sound input, proprioceptive information is required for precise positioning when speaking. To summarize the above studies, 
Table 2 The effect of speech therapy intervention tested by repeated measures ANOVA

\begin{tabular}{|c|c|c|c|c|c|c|c|}
\hline Source & $\begin{array}{l}\text { Type III sum } \\
\text { of squares }\end{array}$ & df & $\begin{array}{l}\text { Mean } \\
\text { square }\end{array}$ & $\mathbf{F}$ & $P$ value & $\begin{array}{l}\text { Partial eta } \\
\text { squared }\end{array}$ & $\begin{array}{l}\text { Observed } \\
\text { power }^{\mathrm{a}}\end{array}$ \\
\hline \multicolumn{8}{|l|}{ Within-subjects effect } \\
\hline Pre/post-ST & 405.600 & 1 & 405.600 & 70.393 & 0.000 & 0.715 & 1.000 \\
\hline Pre/post-ST $\times$ group & 64.067 & 1 & 64.067 & 11.119 & 0.002 & 0.284 & 0.896 \\
\hline Error & 161.333 & 28 & 5.762 & & & & \\
\hline
\end{tabular}

Note: a Computed using alpha $=0.05$.

Abbreviations: ANOVA, analyses of variance; ST, speech therapy.

the developmental process of sensory integration includes the development of articulation. Cermak et $\mathrm{a}^{12}$ found that children with articulation disorders had more concomitant problems in motor coordination than normal children of the same age. Amorosa et $\mathrm{a}^{11}$ also suggested that children with articulation disorders often have concomitant motor coordination problems. A study by Zhao et al ${ }^{29}$ suggested that children with functional articulation disorders are prone to have concomitant ADHD, which produces problems such as emotional misbehavior. Children with SID also display this phenomenon. From the above studies, it is reasonable to assume some correlation between functional articulation disorder and SID.

Our study showed that the differences in pre- and posttherapy number of mistakes in pronunciation between the two groups were significant $(P<0.001)$, indicating a significant difference in efficacy between the two groups. The effect size indicated that the no-SID group made more improvement than the SID group, and the difference between the two groups was very large. These results support our hypothesis that speech therapy would be less efficacious in children with SID than in those without SID. This finding highlights the importance to subjects and caregivers of identifying SID before intervention.

\section{Recommendations for future studies and limitations}

A major limitation of this study is the small sample size, which affects generalization of study results. Future studies with large sample sizes are needed to confirm our findings. In addition, we did not record the content of the other treatments (eg, occupational therapy) in this study. The influences of concomitant psychiatric or attention disorder (ADHD), and the other treatments could not be explored in this study. Finally, future research could seek to answer the following questions: Would speech therapy incorporating a sensory integration approach potentially lead to greater improvement in subjects with SID? What are the long-term outcomes of the two groups?

\section{Conclusion}

Speech therapy improved the articulation performance of children with functional articulation disorders whether or not they also had SID, but it resulted in significantly greater improvement in children without SID. SID may affect the treatment efficiency of speech therapy in young children with articulation disorders. Identification of concomitant SID may prove important to predicting the efficacy of speech therapy.

\section{Acknowledgments}

We thank the children, their parents, and caregivers for their participation in our study, and the therapists who kindly provided assistance with data collection.

\section{Disclosure}

All authors state that they have no commercial, financial or personal relationships which could lead to a conflict of interest that could inappropriately influence (bias) their work.

\section{References}

1. Jacqueline BW. Articulatory and Phonological Impairments. Boston, MA: Allyn \& Bacon; 2000.

2. Sharp HM, Hillenbrand K. Speech and language development and disorders in children. Pediatr Clin North Am. 2008;55(5):1159-1173.

3. Gierut JA. Treatment efficacy: Functional phonological disorders in children. J Speech Hear Res. 1998;41(1):S85-S100.

4. Shriberg LD, Kwiatkowski J. Developmental phonological disorders I: A clinical profile. J Speech Hear Res. 1994;37:1100-1126.

5. Chen HY, Cheng HC, Cherng RJ. Concomitant motor impairments in children with developmental speech and language disorders. Formos J Phys Ther. 2009;34(6):383-393. Article in Chinese.

6. Schaaf RC, Miller LJ. Occupational therapy using a sensory integrative approach for children with developmental disabilities. Ment Retard Dev Disabil Res Rev. 2005;11(2):143-148.

7. Ayres AJ. Sensory Integration and Learning Disorders. Los Angeles, CA: Western Psychological Services; 1991.

8. Bundy AC, Lane SJ, Murray EA. Sensory Integration: Theory and Practice, 2nd ed. Philadelphia, PA: FA. Davis; 2002.

9. Takarae Y, Luna B, Minshew NJ, Sweeney JA. Patterns of visual sensory and sensorimotor abnormalities in autism vary in relation to history of early language delay. J Int Neuropsychol Soc. 2008;14(6):980-989.

10. McDonald ET. A Deep Test of Articulation. Pittsburgh, PA: Stanwix House; 1964.

11. Amorosa H, Benda UV, Dames M, Schaferskupper P. Deficits in fine motor coordination in children with unintelligible speech. Eur Arch Psychiatry Neurol Sci. 1986;236(1):26-30. 
12. Cermak SA, Ward EA, Ward LM. The relationship between articulation disorders and motor coordination in children. Am J Occup Ther. 1986;40(8):546-550.

13. Iwanaga R, Ozawa H, Kawasaki C, Tsuchida R. Characteristics of the sensory-motor, verbal and cognitive abilities of preschool boys with attention deficit/hyperactivity disorder combined type. Psychiatry Clin Neurosci. 2006;60(1):37-45.

14. Allison CL, Gabriel H, Schlange D, Fredrickson S. An optometric approach to patients with sensory integration dysfunction. Optometry. 2007;78(12):644-651.

15. Qvarnstrom MJ, Laine MT, Jaroma SM. Prevalence of articulatory disorders of different sounds in a group of Finnish first-graders. J Commun Disord. 1991;24(5-6):381-392.

16. Wang TM, Liao HF. Assessment accuracy and cut-off points of Comprehensive Developmental Inventory for Infants and Toddlers (CDIIT). Bull Spec Educ. 2007;32(1):1-15. Article in Chinese.

17. Lin CK. The development of the sensory integration functions assessment scale. Psychol Test. 2010;57(3):403-432. Article in Chinese.

18. Lin CL, Min YF, Chou LW, Lin CK. Effectiveness of sensory processing strategies on activity level in inclusive preschool classrooms. Neuropsychiatr Dis Treat. 2012;8:475-481.

19. Jeng JY. The inconsistency of Mandarin consonant production in the different rime contexts for the preschool children. J Speech-Lang-Hear Assoc Taiw. 2009;24(1):59-78. Article in Chinese.

20. Oetter P, Richter EW, Frick SM. MORE: Integrating the Mouth with Sensory and Postural Functions, 2nd ed. Stillwater, MN: PDP Press; 1995.
21. Rvachew S, Nowak M, Cloutier G. Effect of phonemic perception training on the speech production and phonological awareness skills of children with expressive phonological delay. Am J Speech Lang Pathol. 2004;13(3):250-263.

22. Forrest K, Iuzzini J. A comparison of oral motor and production training for children with speech sound disorders. Semin Speech Lang. 2008;29(4):304-311.

23. Rvachew S, Nowak M. The effect of target-selection strategy on phonological learning. J Speech Hear Res. 2001;44(3):610-623.

24. Clark HM. The role of strength training in speech sound disorders. Semin Speech Lang. 2008;29(4):276-283.

25. Koegel RL, Camarata S, Koegel LK, Ben-Tall A, Smith AE. Increasing speech intelligibility in children with autism. J Autism Dev Disord. 1998;28(3):241-251.

26. Williams GC, McReynolds LV. The relationship between discrimination and articulation training in children with misarticulations. $J$ Speech Hear Res. 1975;18(3):401-412.

27. Ayres AJ. Sensory Integration and the Child. Los Angeles, CA: Western Psychological Services; 2005

28. Nasir SM, Ostry DJ. Somatosensory precision in speech production. Curr Biol. 2006;16(19):1918-1923.

29. Zhao YJ, Sun HW, Ma HW, Zhao YR. Comorbidities and behavioral problems in children with functional articulation disorder. Zhongguo Dang Dai Er Ke Za Zhi. 2009;11(3):225-228. Article in Chinese.
Neuropsychiatric Disease and Treatment

\section{Publish your work in this journal}

Neuropsychiatric Disease and Treatment is an international, peerreviewed journal of clinical therapeutics and pharmacology focusing on concise rapid reporting of clinical or pre-clinical studies on a range of neuropsychiatric and neurological disorders. This journal is indexed on PubMed Central, the 'PsycINFO' database and CAS.

\section{Dovepress}

The manuscript management system is completely online and includes a very quick and fair peer-review system, which is all easy to use. Visit $\mathrm{http}: / / \mathrm{www}$.dovepress.com/testimonials.php to read real quotes from published authors. 